\title{
Genome-wide Analysis and Expression Profiling Suggest Diverse Roles of TCP Genes During Development and Stress Responses in Grapevine (Vitis vinifera $\mathrm{L}$ )
}

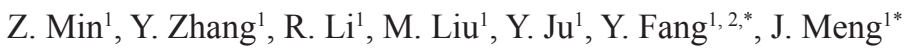 \\ (1) College of Enology, Northwest A\&F University, Yangling, Shaanxi, China \\ (2) Shaanxi Engineering Research Center for Viti-Viniculture, Yangling, Shaanxi, China
}

Submitted for publication: December 2017

Accepted for publication: April 2018

Key words: Genome-wide analysis, TCP transcription factor, grapevine, stress, real-time PCR

\begin{abstract}
Teosinte branched 1/cycloidea/proliferating cell factor 1 (TCP) proteins are plant-specific transcription factors playing crucial roles in various biological processes, such as leaf development, flower symmetry, shoot branching and senescence. However, no comprehensive analysis of the TCP gene family has been reported in grapevine (Vitis vinifera $\mathrm{L}$ ). Herein, a total of 15 TCP family members were identified in the genome of grapevine, located on eight of the 19 chromosomes. Phylogenetic and structural analyses showed that the VvTCPs were classified into two groups, designated as Class I and Class II. The Class II genes were further divided into two subclasses, the CIN subclass and the CYC/TB1 subclass. Genes belonging to the same subclass shared similar gene structures, conserved domains and motifs. Real-time PCR showed that almost all members of Class II exhibited organ-specific expression patterns, while members of Class I and the CIN Class were ubiquitously expressed in all the tissues examined, indicating multiple roles in the development of different grapevine organs. In addition, many members were strongly modulated by abiotic (cold, heat, drought) and biotic (downy mildew and powdery mildew infection) stresses, suggesting important and diverse regulatory roles in adverse conditions and plant immunity. The comprehensive in silico analysis of the grapevine TCP transcription factor family gives us some references to potential functions in grapevine development and stress responses.
\end{abstract}

\section{INTRODUCTION}

The teosinte branched 1 /cycloidea/proliferating cell factor 1 (TCP) gene family is a plant-specific transcription factor, which is of great importance in the evolution and developmental control of plants (Navaud et al., 2007). It was first described in 1999 and named after the first four characterised family members: TEOSINTE BRANCHED 1 (TB1) in maize (Zea mays), CYCLOIDEA (CYC) in snapdragon(Antirrhinum majus), and two PROLIFERATING CELL FACTORS (PCF1 and PCF2) in rice (Oryza sativa) (Doebley et al., 1995; Luo et al., 1996; Kosugi \& Ohashi, 1997; Cubas et al., 1999). All the TCP family members share a conserved region consisted of 59 residue-long amino acids, designated as the TCP domain (Cubas et al., 1999), which form a basic helix-loop-helix (bHLH) structure and are essential for DNA binding and protein interactions (Cubas et al., 1999). Based on differences within the TCP domains, TCP family members were classified into two subfamilies, known as Class I (or PCF or TCP-P) and Class II (or TCP-C) (Navaud et al., 2007), which have been suggested to play contrasting roles in plant proliferation and growth (Hubbard et al., 2002; Takeda et al., 2003; Aguilar-Martínez et al., 2007; Hervé et al., 2009; Martin-Trillo \& Cubas, 2010). TCP Class II was further subdivided into the CIN and CYC/ TB1 clades (Martin-Trillo \& Cubas, 2010). Compared to that of Class I, the TCP domain within the Class II members possessed a four amino acid insertion, and some of the members in TCP Class II possessed an R domain (an 18- to 20-residue arginine-rich motif) and an ECE motif (a glutamic acid-cysteine-glutamic acid stretch), although both were of unknown biological function (Navaud et al., 2007; MartinTrillo \& Cubas, 2010).

TCP transcription factors were reported to be implicated in a series of developmental processes, including flower organ morphogenesis (Damerval et al., 2007; Broholm et al., 2008; Tanaka et al., 2011), the circadian clock (Giraud et al., 2010), leaf shape (Kieffer et al., 2011), internode length (Kieffer et al., 2011), root hair elongation (Hao et al., 2012), cotton fibre elongation (Hao et al., 2012) and tendril development (Wang et al., 2015b). Also, an increasing number of TB1 homologs were identified as negative regulator of axillary

*Corresponding author: E-mail address: mjfwine@nwafu.edu.cn;fangyulin@nwsuaf.edu.cn

Acknowledgments: This work was financially supported by the National Natural Science Foundation of China (Grant No. 31872049), the Key Research and Development Program of Ningxia (2016BZ0602), and the China Agriculture Research System for Grape (CARS-29-zp-6) 
bud outgrowth and tillering (Takeda et al., 2003; AguilarMartínez et al., 2007; Martín-Trillo et al., 2011; Xu et al., 2015). In addition, TCP15 in Arabidopsis was recently found to play roles in modulating redox development and regulating anthocyanin biosynthesis (Viola et al., 2016). TCP factors not only act as mediators of hormone-induced changes in cell proliferation, but also as modulators, or even key players, of hormone synthesis, transport and signal transduction (reviewed in Nicolas \& Cubas, 2016).

To date, many TCP genes have been identified in various plants, such as Arabidopsis thaliana (Yao et al., 2007), Oryza sativa (Yao et al., 2007), Malus domestica (Xu et al., 2014), Solanum lycopersicum (Parapunova et al., 2014), Citrullus lanatus (Shi et al., 2016), Gossypium raimondii (Ma et al., 2014) and Gossypium arboretum (Ma et al., 2016), etc. The sequenced genome of grapevine (Jaillon et al., 2007), and the rise of next-generation sequencing, have made it easier for genome-wide analysis of all genes belonging to specific gene families. The genome-wide analysis of the NAC (Wang et al., 2013), bZIP (Liu et al., 2014), WRKY (Wang et al., 2014), MADS-box (Grimplet et al., 2016) and HSF (Hu et al., 2016) gene families in grapevine have been reported successively. However, no report on the genomewide analysis of the grapevine TCP gene family is currently available.

In this study, we performed for the first time a comprehensive analysis of the TCP gene family members in grapevine (Vitis vinifera L) genome. Next, the phylogenetic analysis, chromosomal location, gene structure and conserved motif were carefully analysed. Finally, the expression profiles in different grapevine tissues and the expression patterns in response to abiotic (heat, cold and drought treatment) and biotic (downy mildew and powdery mildew infection) stimuli were detected via real-time PCR. This study provides valuable information for understanding the classification and molecular and biological functions of VvTCPS, which can be of great value in Vitis breeding.

\section{MATERIALS AND METHODS}

Identification of TCP genes in grapevine (Vitis vinifera $\mathrm{L}$.) The protein sequences of the 15 putative grapevine TCP members available in the Plant Transcription Factor Database (http://planttfdb.cbi.pku.edu.cn/) (Jin et al., 2017) were used as queries to perform BLAST-P (Protein Basic Local Alignment Search Tool) searches in NCBI (National Center for Biotechnology Information, http://blast.ncbi.nlm.nih. gov/Blast.cgi), setting the threshold of the E-value as 1e10 to ensure the discovery of all potential TCP-type DNAbinding domain-encoding sequences in the grape genome (V. vinifera cv. Pinot Noir, PN40024, 12X) (http://www. genoscope.cns.fr/externe/GenomeBrowser/Vitis/). Then, the candidate TCP proteins were manually inspected with the InterProScan program (http://www.ebi.ac.uk/Tools/pfa/iprscan5/) (Quevillon et al., 2005) to validate the presence and completeness of the TCP domain (PF03634). The multiple alignment of identified gene sequences was also performed by MEGA 5.0 (Tamura et al., 2011) to remove redundant sequences.
Chromosomal location and gene structure analysis of VvTCP genes

Molecular weight and isoelectric point were predicted using the ProtParam tool (http://web.expasy.org/protparam/) (Gasteiger et al., 2005); chromosomal locations of the TCP genes were retrieved form the Grape Genome Browser (12X) (http://www.genoscope.cns.fr/externe/GenomeBrowser/ Vitis/), and the distribution of genes in chromosomes were depicted based on the above results. Gene structures were analysed and drawn with the GSDS (Gene Structure Display Server: http://gsds.cbi.pku.edu.cn) (Hu et al., 2015) by aligning the genome sequences with their corresponding coding sequences.

\section{Sequence alignment and phylogenetic analysis}

DNAMAN 6.0 software (Lynnon Biosoft, Quebec, Canada) was employed to perform multiple sequence alignments, with the parameters setting as default. The TCP amino acid sequences of Arabidopsis were downloaded from the Plant Transcription Factor Database, and a phylogenetic tree containing the TCP amino acid sequences of Arabidopsis and grapevine was constructed based on MUSCLE (Edgar, 2004) alignment, as well as the unrooted neighbour-joining method using MEGA 5.0 (Tamura et al., 2011). Neighbourjoining analysis with pairwise deletion was performed using the Jones Taylor Thornton (JTT) model (Jones et al., 1992). Bootstrap analysis was performed with 1000 replicates to assess the level of statistical support for each tree node (Tamura et al., 2011). The protein domains of the putative VvTCPs were identified using MEME online tools (http:// meme.nbcr.net/meme/) (Bailey et al., 2009). Default settings were retained for the parameters.

\section{Plant materials and treatments}

One-year-old 'Cabernet Sauvignon' (Vitis vinifera L) cuttings were planted in plastic pots (diameter: $14 \mathrm{~cm}$, height: $10 \mathrm{~cm}$ ) filled with soil mix (peat:perlite, $4: 1, \mathrm{v}: \mathrm{v}$ ), and grown in the greenhouse of the Enology College, Northwest A\&F University, Yangling, China $\left(34.29^{\circ} \mathrm{N}, 108.35^{\circ} \mathrm{E}\right)$, with the temperature ranging from $22^{\circ} \mathrm{C}$ to $27^{\circ} \mathrm{C}$ and the relative humidity from $70 \%$ to $90 \%$. The roots, stems, leaves (old and young leaves), tendrils, buds (prompt and latent buds), and inflorescences were collected to analyse the tissue-specific expression of the $V v T C P$ genes.

The extreme temperature treatments were performed by transferring the cuttings to two growth chambers in which the temperature setting was $45^{\circ} \mathrm{C}$ and $4^{\circ} \mathrm{C}$ respectively; another set of cuttings was maintained under the control temperature of $23^{\circ} \mathrm{C}$. The drought treatment was simulated by adding Hoagland solution (Hoagland \& Arnon, 1950) with $10 \%$ PEG; another set of cuttings was cultivated similarly with Hoagland solution (Hoagland \& Arnon, 1950). All the leaves were collected $12 \mathrm{~h}$ after the treatments. All of the experiments were repeated independently in triplicate. The samples were frozen immediately in liquid nitrogen and then stored at $-80^{\circ} \mathrm{C}$ for further experiments.

\section{RNA extraction and real-time PCR}

Total RNA was extracted from the materials described above using Plant RNAout (TIANDZ, China) according to the 
manufacturer's instructions. Genomic DNA contamination was removed from the total RNA using RNase-free DNase I (Thermo Fisher Scientific Inc., USA). RNA concentration and quality were assessed using the NanoDrop ND-2000 Spectrophotometer (Thermo Fisher Scientific Inc., USA). The first strand of cDNA was synthesised from RNA using the RevertAid First Strand cDNA Synthesis Kit (Thermo Fisher Scientific Inc., USA). A total of $20 \mu \mathrm{l}$ of qPCR mixture was employed, containing $2 \mu \mathrm{l}$ first-strand cDNAs, $0.6 \mu \mathrm{l}$ of the forward and reverse primers for each gene, $6.8 \mu \mathrm{l}$ of $\mathrm{ddH}_{2} \mathrm{O}$ and $10 \mu \mathrm{l}$ of $2 \times$ FastStart Universal SYBR Green Master (Roche, Germany). The relative expression of each $T C P$ gene was normalised using the method of $2^{-\Delta \Delta \mathrm{Ct}}$ against the reference gene, $V v G A P D H$. The primers were designed via the Primer Premier 5.0 (Lalitha, 2000) and are listed in Table 1.

\section{RESULTS}

\section{Identification of TCP genes in the grapevine genome}

Fifteen TCP proteins were identified in the transcription factor database. A total of 16 homologous TCP proteins were obtained using the BLAST-P in NCBI, 15 of which were detected to contain TCP domain, while others were the repetitive sequences or the alternative splicing transcripts of the TCP genes identified in the transcription factor database. Therefore, as was predicted in the plant transcription factor database, there are 15 members of TCP transcription factors in the grapevine genome (Table 2). According to the chromosomal locations, these putative grapevine (Vitis vinifera $\mathrm{L}$ ) TCP genes were provisionally named $V v T C P 1$ to $V v T C P 15$. As described in Table 2, these grapevine TCPs had a nucleotide length ranging from 654 ( $V v T C P 13)$ to
1398 bp ( $V v T C P 9)$, a peptide length ranging from 217 to 465 amino acids, a molecular weight ranging from 24.7374 to $52.1817 \mathrm{kDa}$, and a theoretical isoelectric point that varied from 6.9057 (VvTCP4) to 10.7992 (VvTCP5).

According to the genomic data, we depicted the chromosome location of all the $V v T C P$ s, finding that these genes were not evenly distributed over the genome of Vitis vinifera $\mathrm{L}$. They were located on Chr 1, Chr 2, Chr 10, Chr 12, Chr 14, Chr 15, Chr 17 and Chr 19. Chr 14 and Chr 17 both had three TCP genes, and Chr 1, Chr 10 and Chr 12 all had two genes (Fig. 1).

\section{Phylogenetic analysis of TCP proteins}

To shed light on the evolutionary relationships of the TCP transcription factor family in grapevine, the fulllength protein sequences of $15 V v T C P s$ and 24 TCPs from Arabidopsis (Yao et al., 2007) were subjected to a multiple sequence alignment using the MEGA 5.0 program. The multiple sequence alignment file was then used to construct an unrooted phylogenetic tree. As illustrated in the neighbourjoining phylogenetic tree (Fig. 2), the $V v T C P S$ were also classified into two groups, designated Class I (TCP-P or PCF class) and Class II (TCP-C). The Class II genes were further classified into two subgroups, CYC/TB1 and CIN. Class I and Class II included six and nine genes respectively, and the group of CYC/TB1 was the smallest one and was consistent with that of Arabidopsis, rice (Oryza sativa) (Yao et al., 2007) and apple (Malus domestica) (Xu et al., 2014). It was also suggested that grapevine TCP members in each group all had their counterparts in Arabidopsis, and they were also not evenly distributed in each group. In addition, only two sister pairs of paralogous $V v T C P$ genes $(V v T C P 2$ and

TABLE 1

Primers used in real-time PCR

\begin{tabular}{lll}
\hline Gene name & Forward primer $\left(5^{\prime}{ }^{\prime} 3^{\prime}\right)$ & Reverse primer (5'-3') \\
\hline VvTCP1 & AAGAACAGAAAACCTCGTAGTAAAT & TCAATGATGCCCACAGTAGCCA \\
VvTCP2 & AGAAAGAGGCAGCCAGAGCAGGA & GGCAAGAGAGCCATTGGAGTAGG \\
VvTCP3 & CCATCCATCGCTTGACTCTTTTG & TTATCGTTGGCGTTGTTCTCCTC \\
VvTCP4 & AGTTTGGAGCACGCATCATCG & GAATAGCAGTGTTAGCAGACAATCGC \\
VvTCP5 & CTACCAACAAAACAAAAACACCAAACA & GCTGCCTCTGGGAGTCAACAATA \\
VvTCP6 & TCACAGAACTACTCACTGGGGGT & GGGATAAGATGGTCGGGAACAAA \\
VvTCP7 & TGGCTAATCAAGAAGGCAAAAT & GTGTCAGCAATGGAATCAGAGT \\
VvTCP8 & CCTCCCTCGCCATCGCTA & GACTTGTGCCCCAACTCTCTCG \\
VvTCP9 & CTGAGGGCTAAGTCAAAGGAAGT & CAAATGATAGTAAGGACCATAAGGC \\
VvTCP10 & AGGGCAAGGGAAAGGACAAG & GAGGTGGGTGGGGAAAATAGC \\
VvTCP11 & GGCACACGAAGGTAGAGGGA & CGGAGGAGACTGAAACGGCA \\
VvTCP12 & ATCCCAACTTCTCGGTCCCTCAA & GCCTCTTCCTCCCTATGCTCTCC \\
VvTCP13 & AAGACAGTGGTCAGCATTTAGAA & CTGGAGTGGTGGAAGTTTATCAA \\
VvTCP14 & TCTTCCTTCTTCTACTTTCCGTCTC & ATCGGCTCGGTCACACTGTTACTT \\
VvTCP15 & CCCAACCCCAATCCTAACCCTAA & GTGTCCGCAATGGTATCAGAGTC \\
VvGAPDH & ACTGCCTTGCTCCTCTTGCGAAG & CCAGTGCTGCTAGGAATGATGTTGAATG \\
\hline
\end{tabular}




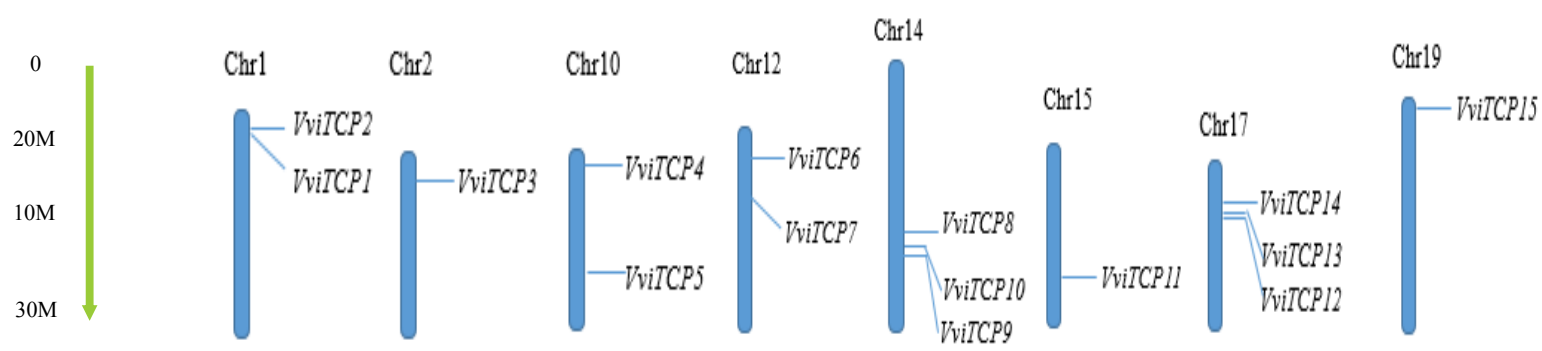

FIGURE 1

The distribution of grapevine TCP gene families in the chromosomes. The location of each TCP gene is indicated by a line.

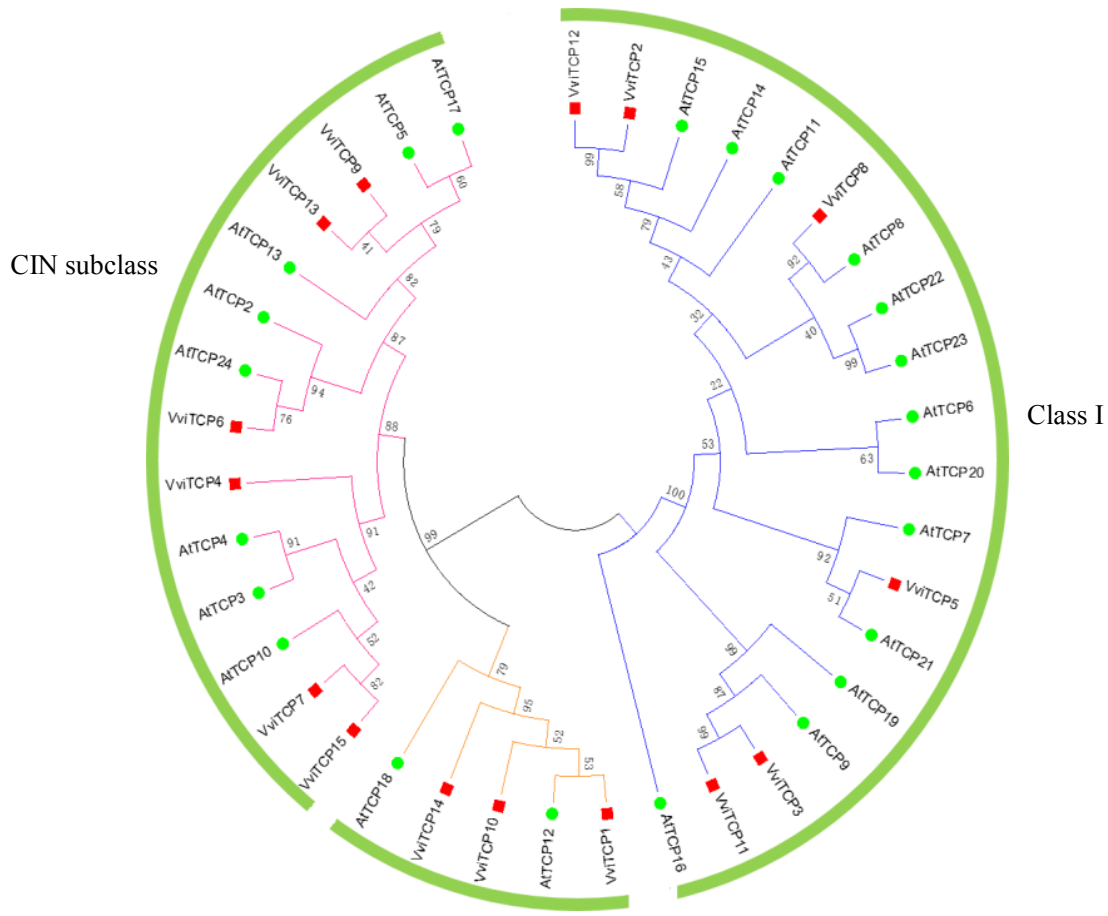

CYC/TB1 subclass

FIGURE 2

Phylogenetic relationships of Arabidopsis and grapevine TCP proteins. The phylogenetic tree was constructed using MEGA 5.0 with the neighbour-joining (NJ) method with 1000 bootstrap replicates.

$V v T C P 2, V v T C P 3$ and $V v T C P 13)$ were identified (Fig. 3), with strong bootstrap support $(>90 \%)$. This may account for the fewer number of TCP members in grapevine than in other species.

\section{Conserved domains and gene structure analysis of TCP gene family in grapevine}

The sequence alignment analysis of these TCP proteins showed that the specific TCP domain of bHLH was highly conserved in all the members. However, the $\mathrm{R}$ domain, an 18- to 20-residue arginine-rich motif, was only present in the Class II members. A four amino acid deletion was observed in Class II (Fig. 3). In addition, the sequence conservation outside the TCP domain was low, and sequence lengths on both sides of the TCP domain varied greatly among the
15 members. This result was mainly consistent with the phylogenetic analysis described above.

Gene structure was analysed and drawn with the GSDS (http://gsds.cbi.pku.edu.cn) by aligning the genome sequences with their corresponding CDS sequences. As was shown in Fig. 4, most TCP members (14 members, 93.3\%) contained both exons and introns, except for one member, VvTCP5, which had no intron. Niu and Yang (2011) reported that highly expressed genes had higher intron densities (number of introns per kilobase of coding sequence) than weakly expressed genes, and introns could mediate the enhancement of transcription by splicing. As in other species, members of Class II had more introns than those of Class I, indicating a higher level of regulation by introns. Six genes $(40 \%)$ had two introns, three genes $(20 \%)$ had three introns, 


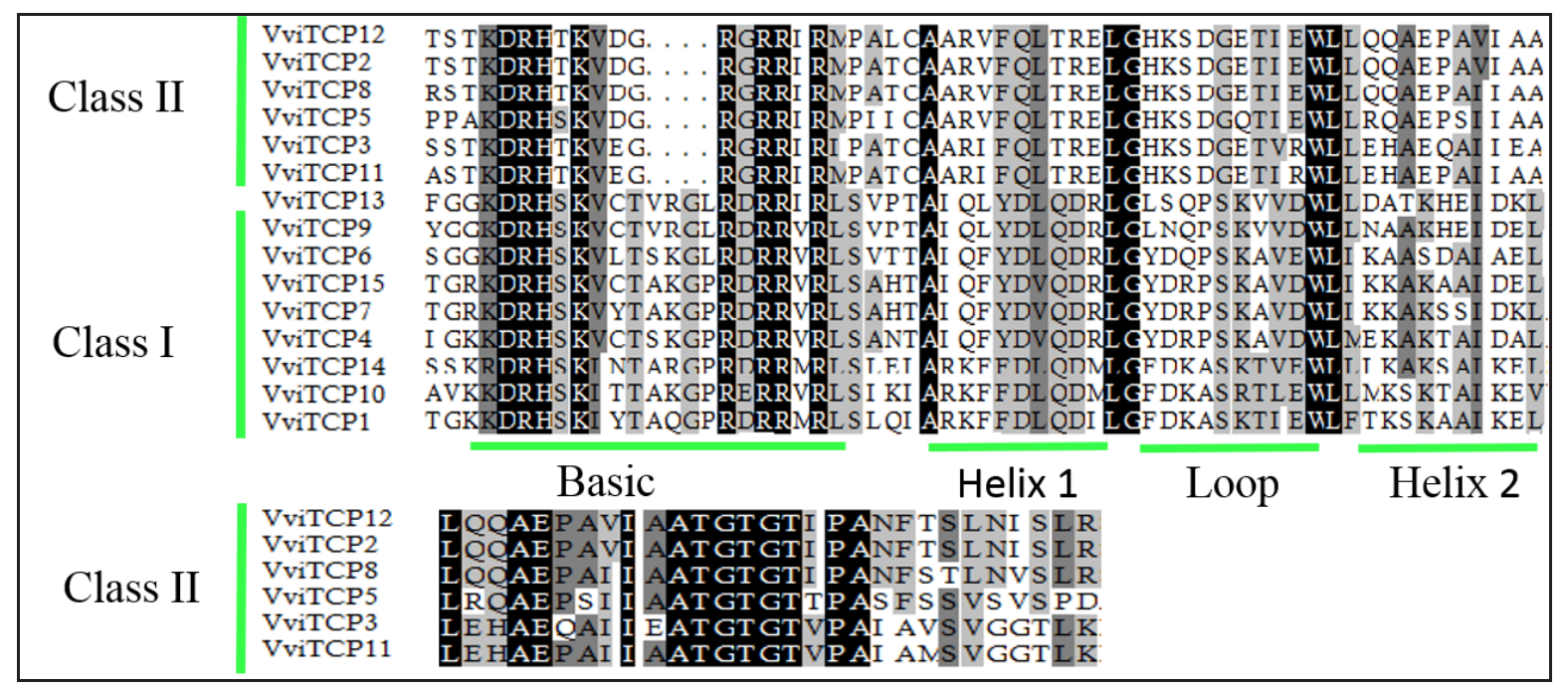

FIGURE 3

Alignment of amino acid sequences among grapevine TCP members.

TABLE 2

Characteristics of the TCP transcription factor family in Vitis vinifera $\mathrm{L}$

\begin{tabular}{|c|c|c|c|c|c|c|}
\hline Name & TF ID & Chromosome location & $\begin{array}{l}\text { Nucleotide } \\
\text { length (bp) }\end{array}$ & $\begin{array}{l}\text { Amino } \\
\text { acid } \\
\text { length }\end{array}$ & $\begin{array}{l}\text { Molecular } \\
\text { weight } \\
(\mathrm{kDa})\end{array}$ & $\begin{array}{l}\text { Isoelectric } \\
\text { point }\end{array}$ \\
\hline VvTCP1 & GSVIVT01011962001 & $\operatorname{chr} 1: 2574244 . .2575738$ & 1215 & 404 & 44.8869 & 10.3531 \\
\hline VvTCP2 & GSVIVT01020011001 & $\operatorname{chr} 1: 11610081 . .11611375$ & 954 & 317 & 35.1046 & 9.8435 \\
\hline VvTCP3 & GSVIVT01019876001 & $\operatorname{chr} 2: 4139671 . .4141512$ & 1296 & 431 & 46.746 & 7.9585 \\
\hline VvTCP4 & GSVIVT01021167001 & $\operatorname{chr} 10: 2112286 . .2113434$ & 1149 & 382 & 42.3984 & 6.9057 \\
\hline VvTCP5 & GSVIVT01026145001 & $\operatorname{chr} 10: 12936187 . .12943830$ & 867 & 288 & 30.5565 & 10.7992 \\
\hline VvTCP6 & GSVIVT01021528001 & chr10:6666048..6669048 & 960 & 319 & 34.8686 & 9.7124 \\
\hline VvTCP7 & GSVIVT01020666001 & $\operatorname{chr12:3280522..3283144}$ & 924 & 307 & 33.6998 & 6.9128 \\
\hline VvTCP8 & GSVIVT01023283001 & 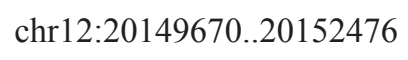 & 1164 & 387 & 42.3781 & 8.0579 \\
\hline VvTCP9 & GSVIVT01032911001 & $\operatorname{chr} 14: 24045544 . .24048408$ & 1398 & 465 & 52.1817 & 9.0525 \\
\hline VvTCP10 & GSVIVT01036449001 & chr14:22124290..22125983 & 822 & 273 & 31.2935 & 10.0656 \\
\hline VvTCP11 & GSVIVT01027588001 & $\operatorname{chr} 15: 15268031 . .15269562$ & 1023 & 340 & 36.3764 & 9.5841 \\
\hline VvTCP12 & GSVIVT01008023001 & chr17:6588505..6590555 & 1038 & 345 & 38.089 & 8.3349 \\
\hline VvTCP13 & GSVIVT01008109001 & $\operatorname{chr} 17: 5711518 . .5719116$ & 654 & 217 & 24.7374 & 9.719 \\
\hline VvTCP14 & GSVIVT01008234001 & $\operatorname{chr} 17: 4343550 . .4345620$ & 1101 & 366 & 41.5708 & 8.9711 \\
\hline VvTCP15 & GSVIVT01014236001 & chr19:1804594..1806793 & 903 & 300 & 32.9047 & 7.5709 \\
\hline
\end{tabular}

only one $(6.7 \%)$ had four introns, two $(13 \%)$ contained six introns and only one $(6.7 \%)$ contained seven introns. Among all the members, $V v T C P 12$ was the longest gene, with an intron region of nearly $6 \mathrm{~Kb}$. Generally speaking, genes belonging to the same subclades had a similar number of exons (Fig. 4).
Conserved motifs analysis of TCP gene family in grapevine

We analysed the motif compositions of the 15 proteins in Vitis vinifera $\mathrm{L}$ using the MEME online tool and found that the number of motifs in grapevine TCP families varied from two to seven (Fig. 4). All the VvTCPs had motif 1 and motif 2, which were specific to the TCP domain, and only the members of Class I had motif 3. Some motifs, such as motif 


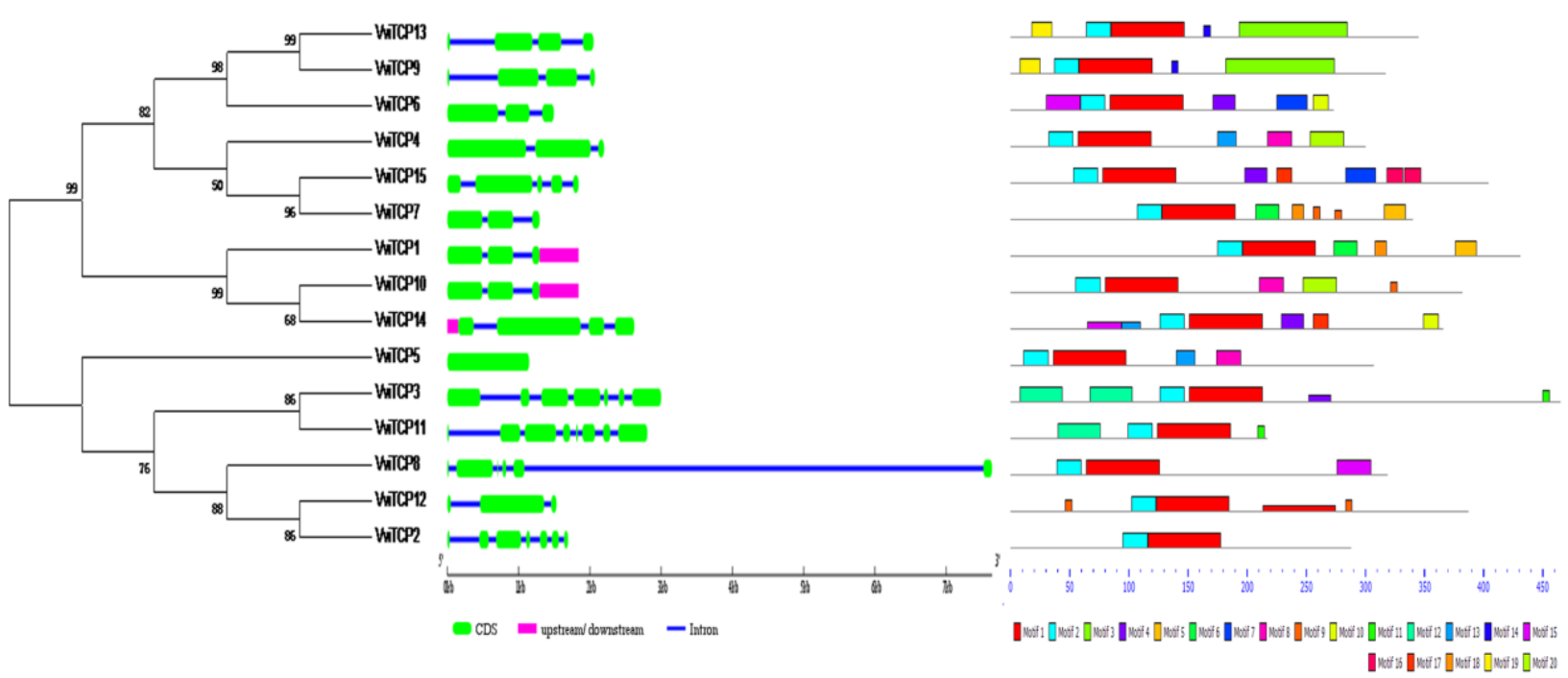

FIGURE 4

Phylogenetic analysis and gene structure of TCP genes in grapevine. The phylogenetic tree was constructed using MEGA 5.0 with the neighbour-joining (NJ) method with 1000 bootstrap replicates. The green and red boxes represent exons and upstream/ downstream sequences respectively, and the blue lines represent introns.

4 , motif 5 and motif 7, existed exclusively in the group of Class I, while others, like motif 12, were present only in the group of Class II. In summary, members in the same subgroup showed the same motif composition and distribution.

\section{Expression profiles of VvTCP genes in different organs and under different treatments}

Real-time PCR was used to detect the expression patterns in different tissues in grapevine, and under different treatments. As shown in Fig. 5, all the members of $V v T C P$ were shown to be expressed in the examined tissues. However, tissuespecific expression was also observed, such as $V v T C P 1$ in the inflorescence and latent buds, and VvTCP2 in the stems (Fig. 5). We also found that most of the genes were apparently suppressed or induced when exposed to abiotic and biotic stress (Fig. 6). Among the 15 genes, six ( $V v T C P 1$, VvTCP4, VvTCP8, VvTCP9, VvTCP13, VvTCP14) were upregulated by heat $\left(45^{\circ} \mathrm{C}\right)$, and cold $\left(4^{\circ} \mathrm{C}\right)$ up-regulated six genes (VvTCP4, VvTCP5, VvTCP8, VvTCP12, VvTCP13, $V v T C P 15)$. Intriguingly, most $V v T C P$ gene expression was strongly modulated under the infection of two common but harmful pathogens, namely downy and powdery mildew, implying an additional role in disease responses except for the functions previously reported. Overall, the expression profile analysis indicated that $V v T C P$ genes might play multiple roles in grapevine tissue development and abiotic and biotic stress responses.

\section{DISCUSSION}

The TCP transcription factors were versatile and of great importance to the process of plant growth and development. Family members of TCP genes in Arabidopsis (Arabidopsis thaliana) (Yao et al., 2007), rice (Oryza sativa) (Yao et al., 2007), cotton (Gossypium arboretum and Gossypium raimondii) (Ma et al., 2014, 2016), apple (Malus domestica) (Xu et al., 2014), tomato (Solanum lycopersicum)
(Parapunova et al., 2014), watermelon (Citrullus lanatus) (Shi et al., 2016) and Prunus mume (Zhou et al., 2016) have been identified, and some of the genes have been well characterised, while no comprehensive analysis of grapevine has been reported until now. In our study, 15 TCP members in the grapevine genome were identified. Compared to the other species mentioned above, the number in grapevine is fewer, probably because fewer gene-duplication events occurred.

The phylogenetic tree constructed on the basis of the full length of VvTCP proteins in grapevine and Arabidopsis further validates the previous classification of AtTCPs (Yao et al., 2007). The TCP members in grapevine were divided into two groups, namely Class I and Class II (Fig. 2). Class II was further classified into two subgroups, the CYC/TB1 and the CIN subgroup, in which VvTCP members were unevenly distributed (Fig. 2). The multiple alignment of protein sequences in the VvTCPs was consistent with the results of the phylogenetic tree (Figs 2 and 3). Most members of the same subgroup shared relatively similar gene structure, conserved domain and motifs between grapevine and Arabidopsis (Xu et al., 2014), providing a solid reference for us to explore the functions of the $V v T C P$ s.

The phylogenetic tree indicated that VvTCP12 and VvTCP2 showed high similarity with AtTCP14 and AtTCP15, and real-time PCR showed that these genes were highly expressed in old and young leaves and in inflorescences, suggesting that they may also modulate cell proliferation in the developing leaf blade and in specific floral tissues (Hiratsu et al., 2003; Kieffer et al., 2011). Aguilar-Martínez and Sinha (2013) reported that $T C P$ genes in Class I, such as AtTCP7, AtTCP8, AtTCP22 and AtTCP23, could interact with $\mathrm{S} R D X$ to control cell proliferation in leaf development. $V v T C P 8$ was shown to be $92 \%$ homologous with $A t T C P 8$, suggesting possible functions for $V v T C P 8$ in the regulation of leaf development. TCP24 in Arabidopsis 
VvTCP1

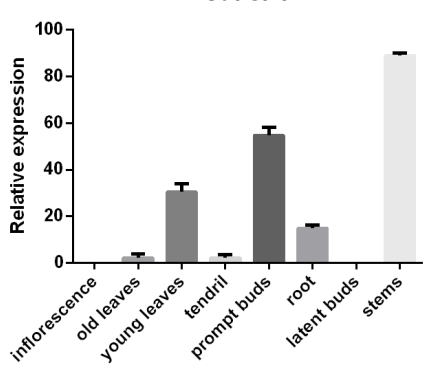

VvTCP5

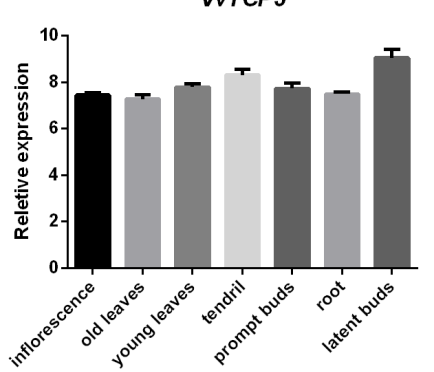

VvTCP9

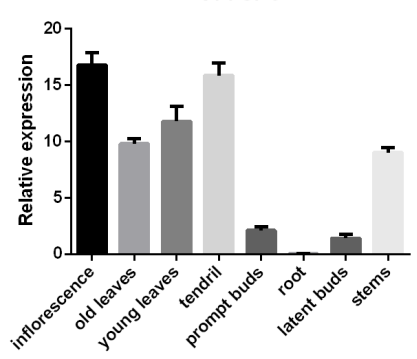

VvTCP13

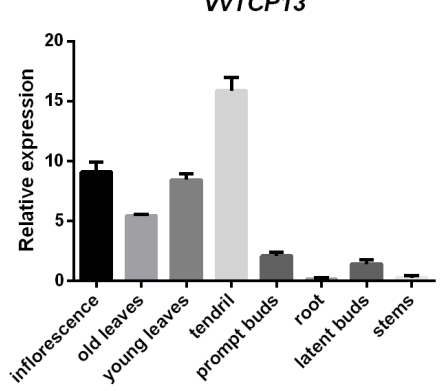

VvTCP2
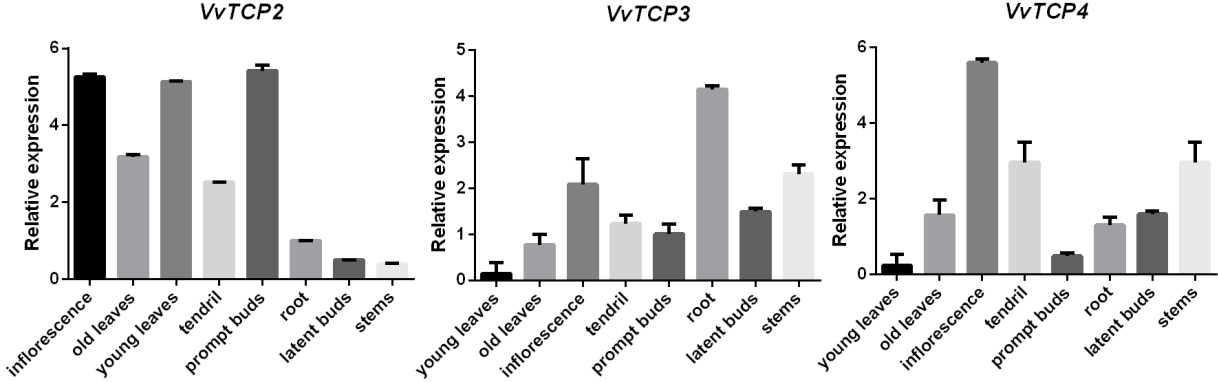

$V_{V T C P 7}$
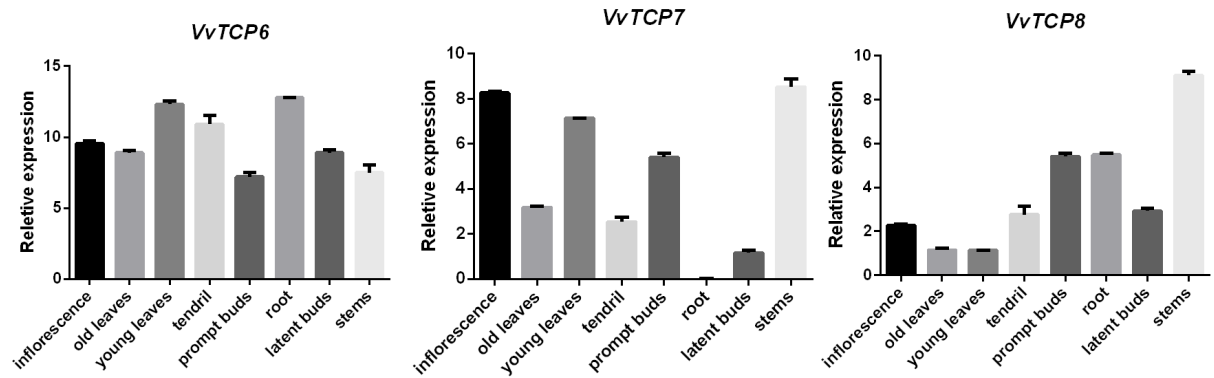

VvTCP11
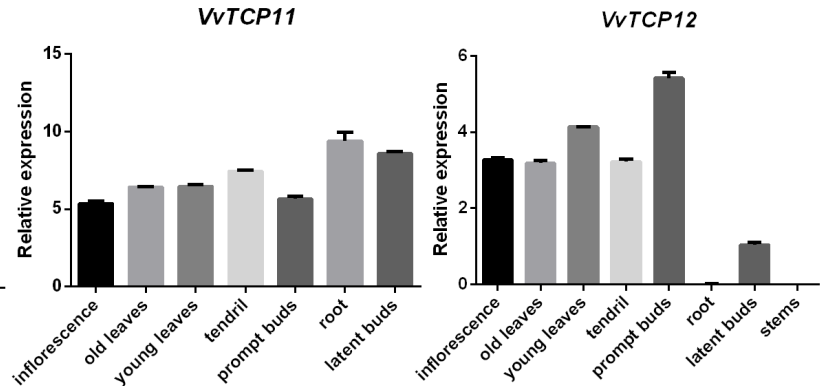

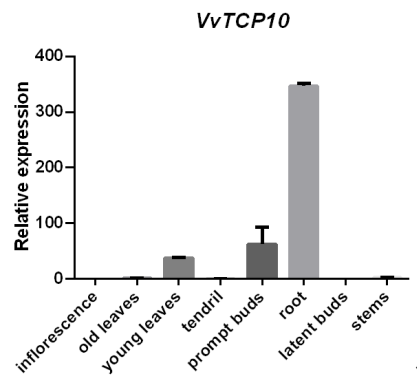

VvTCP14
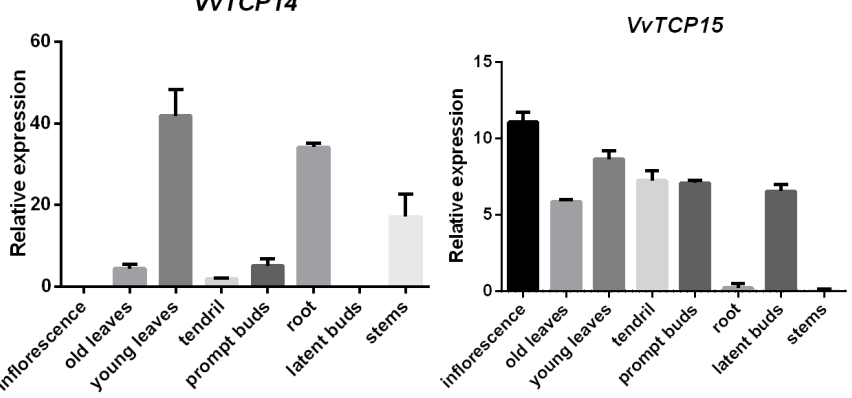

FIGURE 5

Expression patterns of $V v T C P S$ in different grapevine tissues. The Y-axis indicates the relative expression level; the $\mathrm{X}$-axis (inflorescence, old leaves, young leaves, tendrils, prompt buds, root, latent buds, stems) indicates different organs. Standard errors are plotted using vertical lines.

was reported to act as an important regulator of secondary cell wall thickening and to modulate anther endothecium development (Wang et al., 2015a), and VvTCP6 showed 76\% similarity with it, indicating the same functions. In addition, $V v T C P 1, V v T C P 10$ and $V v T C P 14$ were highly homologous to the AtTCP18, which is known as BRANCHEDI(BRC1) and TEOSINTE BRANCHED1-LIKE(TBL1), and could act downstream of cytokinins and strigolactones to coordinate the outgrowth of axillary buds (Aguilar-Martínez et al., 2007; Finlayson, 2007). Although they did not present bud-specific expression (Fig. 5) as in Arabidopsis (AguilarMartínez et al., 2007), they were differentially expressed between latent bud and prompt buds (Fig. 5), which are two kinds of grapevine buds exhibiting different characteristics in terms of dormancy. Therefore, they were identified to be candidates of BRANCHED 1, thus to regulate grapevine bud 

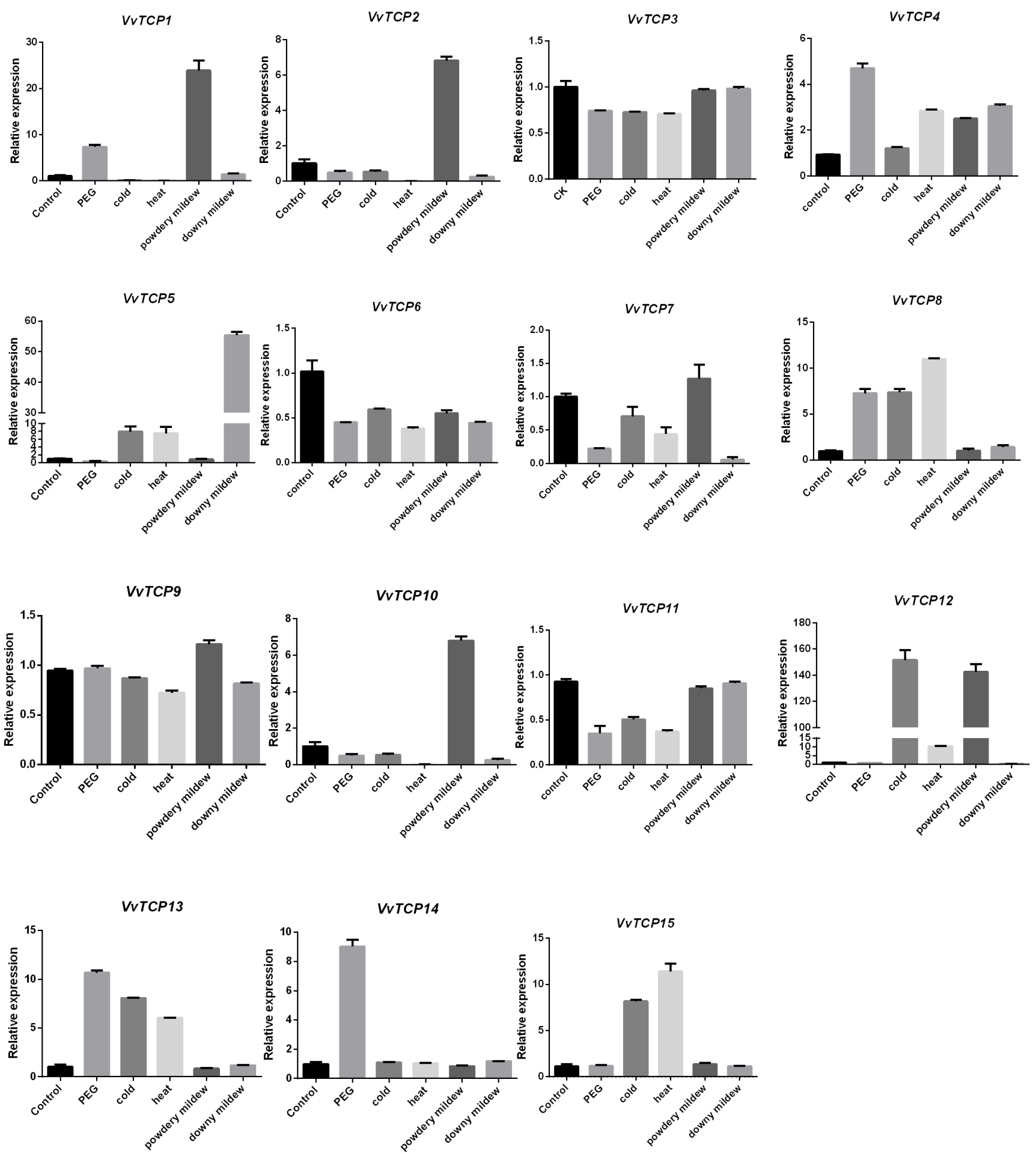

FIGURE 6

Expression patterns of $V v T C P S$ under different abiotic and biotic stresses. The Y-axis indicates the relative expression level; the $\mathrm{X}$-axis (control, PEG, cold, heat, powdery mildew, downy mildew) indicates different treatments. Standard errors are plotted using vertical lines.

break and shoot branching. Interestingly, recent research on grapevine bud burst also identified TCP transcription factors as one of the hallmarks of the orderly and functional resumption of growth following quiescence in buds (Meitha et al., 2018). Hence, the detailed biological functions of TCP transcription factors in grapevine bud break would be an issue worthy of further investigation.
The expression patterns of $15 V v T C P s$ in eight organs were detected via real-time PCR. The results show that almost all the members in Class II had organ-specific expression (Fig. 5), while all members in Class I and the CIN members were ubiquitously expressed in all the tissues examined, further validating their diverse functions in the development of different organs. The divergence between 
the two groups was also consistent with that in other species (Xu et al., 2014; Zhou et al., 2016). Most members, such as $V v T C P 2, V v T C P 5, V v T C P 6, V v T C P 7$ and $V v T C P 14$, had higher expression in young leaves (Fig. 5), implying that they play a role in modulating leaf development.

Plants commonly encounter various abiotic and biotic stresses during their lifecycle. Some studies have reported that TCP factors could act as targets of pathogenic effectors and are likely to play an important role in plant immunity (Lopez et al., 2015). Many TCP members, such as TCP13, $T C P 14, T C P 15, T C P 19$ and TCP21, are reported to be targeted by effectors from three pathogens that span the eukaryote-eubacteria divergence, represented by the bacterium Pseudomonas syringae (Psy), the oomycete Hyaloperonospora arabidopsidis ( $\mathrm{Hpa}$ ), and the true fungus Golovinomyces orontii (Gor) (Mukhtar et al., 2011; Weßling et al., 2014). Deletion mutants of TCP13, TCP14 and TCP19 exhibited enhanced disease susceptibility phenotypes (Mukhtar et al., 2011). In our study, several VvTCPS, including $V v T C P 1, V v T C P 2, V v T C P 10$ and $V v T C P 12$, were highly induced after infection with powdery mildew, and $V v T C P 5$ expression was up-regulated under downy mildew infection, indicating an important regulatory role in plant disease response, providing an important reference in the study of grapevine disease response (Fig. 6).

So far, there have been limited reports on the response of TCP members under abiotic stress. The response of TCPs to abiotic stress in monocots was found to be mediated mainly by abscisic acid (ABA) (Zhou et al., 2013). When exposed to salt, drought and cold stress, OsTCP19 expression in rice was greatly up-regulated, and it could interact with the $A B A$ INSENSITIVE $_{4}\left(\mathrm{ABI}_{4}\right)$, a transcription factor involved in ABA signal transduction (Zhou et al., 2013; Mukhopadhyay et al., 2015). In our study, many TCP genes were also apparently regulated by cold, heat and drought stresses, for example the transcript level of $V v T C P 1, V v T C P 4, V v T C P 8$, $V v T C P 13$ and $V v T C P 14$ was greatly induced by drought treatment (10\% PEG), while six genes (VvTCP1, VvTCP4, $V v T C P 8, V v T C P 9, V v T C P 13, V v T C P 14)$ were up-regulated by heat $\left(45^{\circ} \mathrm{C}\right)$ and six $(V v T C P 4, V v T C P 5, V v T C P 8$, $V v T C P 12, V v T C P 13, V v T C P 15)$ were up-regulated by cold $\left(4^{\circ} \mathrm{C}\right)$, suggesting that all these genes had potential roles to play in response to abiotic stress (Fig. 6). We speculate that, when exposed to adverse conditions, these genes may form homo- or hetero-dimers, and also may interact with other transcription factors to form functional protein complexes, as previously reported in other plant species (Parapunova et al., 2014), to enhance their adaptability to adversity. Further research is needed to validate its role in the response to different stresses.

Overall, the in silico analysis of grapevine TCP transcription factors could provide insight into the potential functions of TCP genes and help to identify some genes associated with different biological processes in important agronomical traits of grapevine, such as bud break, shoot branching, and stress tolerance. This knowledge will support the future improvement of grapevine cultivars via genetic engineering.

\section{CONCLUSIONS}

In this study, 15 unevenly distributed TCP genes were identified on eight chromosomes in the genome of Vitis vinifera L. The $V v T C P$ genes were classified into two groups, as in other species. Real-time PCR indicated that most members of $V v T C P S$ were expressed differentially in different grapevine tissues, and several genes' expression was induced or suppressed under abiotic (heat, cold and PEG) and biotic (downy mildew and powdery mildew infection) stresses, indicating multiple roles in different organs and in various biological processes, especially roles in regulating the process of bud dormancy and modulating plant immunity. To the best of our knowledge, this is the first report on genome-wide analysis of the grapevine TCP gene family, and the first report of its relationship with abiotic and biotic responses. This study provides abundant information for understanding the classification and potential functions of TCP genes in grapevine, which would be of great help to provide guidance in Vitis breeding using genetic engineering tools, and thus be of great significance to the development of the grape industry.

\section{LITERATURE CITED}

Aguilar-Martínez, J.A. \& Sinha, N., 2013. Analysis of the role of Arabidopsis class I TCP genes AtTCP7, AtTCP8, AtTCP22, and AtTCP23 in leaf development. Front. Plant Sci. 4, 406-406.

Aguilar-Martínez, J.A., Poza-Carrión, C. \& Cubas, P., 2007. Arabidopsis $B R A N C H E D 1$ acts as an integrator of branching signals within axillary buds. Plant Cell 19, 458-472.

Bailey, T.L., Boden, M., Buske, F.A., Frith, M., Grant, C.E., Clementi, L., Ren, J., Li, W.W. \& Noble, W.S., 2009. MEME SUITE: Tools for motif discovery and searching. Nucleic Acids Res. 37, 202-208.

Broholm, S.K., Tahtiharju, S., Laitinen, R.A.E., Albert, V.A., Teeri, T.H. \& Elomaa, P., 2008. A TCP domain transcription factor controls flower type specification along the radial axis of the Gerbera (Asteraceae) inflorescence. P. Natl. Acad. Sci. USA 105, 9117-9122.

Cubas, P., Lauter, N., Doebley, J. \& Coen, E., 1999. The TCP domain: A motif found in proteins regulating plant growth and development. Plant J. $18,215-222$.

Damerval, C., Le Guilloux, M., Jager, M. \& Charon, C., 2007. Diversity and evolution of CYCLOIDEA-like TCP genes in relation to flower development in Papaveraceae. Plant Physiol. 143, 759-772.

Doebley, J., Stec, A. \& Gustus, C., 1995. Teosinte branched1 and the origin of maize: Evidence for epistasis and the evolution of dominance. Genetics $141,333-346$.

Edgar, R.C., 2004. MUSCLE: Multiple sequence alignment with high accuracy and high throughput. Nucleic Acids Res 32, 1792-1797.

Finlayson, S.A., 2007. Arabidopsis Teosinte Branched1-like 1 regulates axillary bud outgrowth and is homologous to monocot Teosinte Branched1. Plant Cell Physiol. 48, 667-677.

Gasteiger, E., Hoogland, C., Gattiker, A., Duvaud, S.E., Wilkins, M.R., Appel, R.D. \& Bairoch, A., 2005. Protein identification and analysis tools on the ExPASy server. In: Walker, J.M. (ed). The proteomics protocols handbook. Humana Press, Totowa. $571-607$.

Giraud, E., Ng, S., Carrie, C., Duncan, O., Low, J., Lee, C.P., Van Aken, O., Millar, A.H., Murcha, M. \& Whelan, J., 2010. TCP transcription factors link the regulation of genes encoding mitochondrial proteins with the circadian clock in Arabidopsis thaliana. Plant Cell 22, 3921-3934. 
Grimplet, J., Martínez-Zapater, J.M. \& Carmona, M.J., 2016. Structural and functional annotation of the MADS-box transcription factor family in grapevine. BMC Genomics 17, 1-23.

Hao, J., Tu, L.L., Hu, H.Y., Tan, J.F., Deng, F.L., Tang, W.X., Nie, Y.C. \& Zhang, X.L., 2012. GbTCP, a cotton TCP transcription factor, confers fibre elongation and root hair development by a complex regulating system. J, Exp. Bot. 63, 6267-6281.

Hervé, C., Dabos, P., Bardet, C., Jauneau, A., Auriac, M.C., Ramboer, A., Lacout, F. \& Tremousaygue, D., 2009. In vivo interference with AtTCP20 function induces severe plant growth alterations and deregulates the expression of many genes important for development. Plant Physiol. 149, $1462-1477$

Hoagland, D.R. \& Arnon, D.I., 1950. The water culture method for growing plants without soil. California Agricultural Experiment Station Circular 347.

Hu, B., Jin, J., Guo, A.Y., Zhang, H., Luo, J. \& Gao, G., 2015. GSDS 2.0: An upgraded gene feature visualization server. Bioinformatics 31, 1296.

Hu, Y., Han, Y.T., Zhang, K., Zhao, F.L., Li, Y.J., Zheng, Y., Wang, Y.J. \& Wen, Y.Q., 2016. Identification and expression analysis of heat shock transcription factors in the wild Chinese grapevine (Vitis pseudoreticulata). Plant Physiol. Biochem. 99, 1-10.

Hubbard, L., McSteen, P., Doebley, J. \& Hake, S., 2002. Expression patterns and mutant phenotype of teosinte branched1 correlate with growth suppression in maize and teosinte. Genetics 162, 1927-1935.

Jaillon, O., Aury, J.M., Noel, B., Policriti, A., Clepet, C., Casagrande, A., Choisne, N., Aubourg, S., Vitulo, N. \& Jubin, C., 2007. The grapevine genome sequence suggests ancestral hexaploidization in major angiosperm phyla. Nature 449, 463-467.

Jin, J., Tian, F., Yang, D.C., Meng, Y.Q., Kong, L., Luo, J. \& Gao, G., 2017. PlantTFDB 4.0: Toward a central hub for transcription factors and regulatory interactions in plants. Nucleic Acids Res. 45, D1040-D1045.

Jones, D.T., Taylor, W.R. \& Thornton, J.M., 1992. JTT model: The rapid generation of mutation data matrices from protein sequences. Comput. Appl. Biosci. 8, 275-282.

Kieffer, M., Master, V., Waites, R. \& Davies, B., 2011. TCP14 and TCP15 affect internode length and leaf shape in Arabidopsis. Plant J. 68, 147-158.

Kosugi, S. \& Ohashi, Y., 1997. PCF1 and PCF2 specifically bind to cis elements in the rice proliferating cell nuclear antigen gene. Plant Cell 9, 1607-1619.

Lalitha, S., 2000. Primer premier 5. Biotech Software \& Internet Report 1 270-272.

Liu, J., Chen, N., Chen, F., Cai, B., Santo, S.D., Tornielli, G.B., Pezzotti, M. \& Cheng, Z.M., 2014. Genome-wide analysis and expression profile of the bZIP transcription factor gene family in grapevine (Vitis vinifera). BMC Genomics 15, 1-18.

Lopez, J.A., Sun, Y., Blair, P.B. \& Mukhtar, M.S. 2015. TCP three-way handshake: Linking developmental processes with plant immunity. Trends Plant Sci. 20, 238-245.

Luo, D., Carpenter, R., Vincent, C., Copsey, L. \& Coen, E., 1996. Origin of floral asymmetry in Antirrhinum. Nature 383, 794-799.

Ma, J., Liu, F., Wang, Q.L., Wang, K.B., Jones, D.C. \& Zhang, B.H., 2016 Comprehensive analysis of TCP transcription factors and their expression during cotton (Gossypium arboreum) fiber early development. Sci. Rep. 6, 21535

Ma, J., Wang, Q.L., Sun, R.R., Xie, F.L., Jones, D.C. \& Zhang, B.H., 2014 Genome-wide identification and expression analysis of TCP transcription factors in Gossypium raimondii. Sci. Rep. 4, 6645
Martin-Trillo, M. \& Cubas, P., 2010. TCP genes: a family snapshot ten years later. Trends Plant Sci. 15, 31-39.

Martín-Trillo, M., Grandío, E.G., Serra, F., Marcel, F., Rodríguezbuey, M.L., Schmitz, G., Theres, K., Bendahmane, A., Dopazo, H. \& Cubas, P., 2011. Role of tomato BRANCHED1-like genes in the control of shoot branching. Plant J. 67, 701-714.

Meitha, K., Agudeloromero, P., Signorelli, S., Gibbs, D.J., Considine, J.A., Foyer, C.H. \& Considine, M.J., 2018. Developmental control of hypoxia during bud burst in grapevine. Plant Cell Environ. doi:10.1111/pce.13141

Mukhopadhyay, P., Tyagi, A.K. \& Tyagi, A.K., 2015. OsTCP19 influences developmental and abiotic stress signaling by modulating $\mathrm{ABI}_{4}$-mediated pathways. Sci Rep. 5, 9998.

Mukhtar, M.S., Carvunis, A.R., Dreze, M., Epple, P., Steinbrenner, J., Moore, J., Tasan, M., Galli, M., Hao, T. \& Nishimura, M.T., 2011. Independently evolved virulence effectors converge onto hubs in a plant immune system network. Science 333, 596-601.

Navaud, O., Dabos, P., Carnus, E., Tremousaygue, D. \& Herve, C., 2007. TCP transcription factors predate the emergence of land plants. J. Mol. Evol. 65, 23-33.

Nicolas, M. \& Cubas, P., 2016. TCP factors: New kids on the signaling block. Curr. Opin. Plant Biol. 33, 33-41.

Niu, D.K. \& Yang, Y.F., 2011. Why eukaryotic cells use introns to enhance gene expression: Splicing reduces transcription-associated mutagenesis by inhibiting topoisomerase I cutting activity. Biol. Direct 6, 1-10.

Parapunova, V., Busscher, M., Busscher-Lange, J., Lammers, M., Karlova, R., Bovy, A.G., Angenent, G.C. \& De Maagd, R.A., 2014. Identification, cloning and characterization of the tomato TCP transcription factor family. BMC Plant Biol. 14, 157.

Quevillon, E., Silventoinen, V., Pillai, S., Harte, N., Mulder, N., Apweiler, R. \& Lopez, R., 2005. InterProScan: Protein domains identifier. Nucleic Acids Res. 33, 116-120

Shi, P., Guy, K.M., Wu, W., Fang, B., Yang, J., Zhang, M. \& Hu, Z., 2016. Genome-wide identification and expression analysis of the ClTCP transcription factors in Citrullus lanatus. BMC Plant Biol. 16, 85.

Takeda, T., Suwa, Y., Suzuki, M., Kitano, H., Ueguchi-Tanaka, M., Ashikari, M., Matsuoka, M. \& Ueguchi, C., 2003. The OsTB1 gene negatively regulates lateral branching in rice. Plant J. 33, 513-520.

Tamura, K., Peterson, D., Peterson, N., Stecher, G., Nei, M. \& Kumar, S., 2011. MEGA5: Molecular evolutionary genetics analysis using maximum likelihood, evolutionary distance, and maximum parsimony methods. Mol. Biol. Evol. 28, 2731-2739.

Tanaka, Y., Yamamura, T., Oshima, Y., Mitsuda, N., Koyama, T., OhmeTakagi, M. \& Terakawa, T., 2011. Creating ruffled flower petals in Cyclamen persicum by expression of the chimeric cyclamen TCP repressor. Plant Biotechnol. 28, 141-147.

Viola, I.L., Camoirano, A. \& Gonzalez, D.H., 2016. Redox-dependent modulation of anthocyanin biosynthesis by the TCP transcription factor TCP15 during exposure to high light intensity conditions in Arabidopsis. Plant Physiol. 170, 74-85.

Wang, H., Mao, Y., Yang, J. \& He, Y., 2015a. TCP24 modulates secondary cell wall thickening and anther endothecium development. Front. Plant Sci. 6,436 .

Wang, L., Zhu, W., Fang, L., Sun, X., Su, L., Liang, Z., Wang, N., Londo, J.P., Li, S. \& Xin, H., 2014. Genome-wide identification of WRKY family genes and their response to cold stress in Vitis vinifera. BMC Plant Biol 14, 160-162. 
Wang, N., Zheng, Y., Xin, H., Fang, L. \& Li, S., 2013. Comprehensive analysis of NAC domain transcription factor gene family in Vitis vinifera. Plant Cell Rep. 32, 61-75.

Wang, S.H., Yang, X.Y., Xu, M.N., Lin, X.Z., Lin, T., Qi, J.J., Shao, G.J., Tian, N.N., Yang, Q., Zhang, Z.H. \& Huang, S.W., 2015b. A rare SNP identified a TCP transcription factor essential for tendril development in cucumber. Mol. Plant 8, 1795-1808.

Weßling, R., Epple, P., Altmann, S., He, Y., Yang, L., Henz, S.R., McDonald, N., Wiley, K., Bader, K.C. \& Gläßer, C., 2014. Convergent targeting of a common host protein-network by pathogen effectors from three kingdoms of life. Cell Host \& Microbe 16, 364-375.

Xu, K., Wang, Y., Shi, L., Sun, F., Liu, S. \& Xi, Y., 2015. PvTB1, a Teosinte Branchedl gene homolog, negatively regulates tillering in switchgrass. J. Plant Growth Regul. 35, 44-53.
Xu, R., Sun, P., Jia, F., Lu, L., Li, Y., Zhang, S. \& Huang, J., 2014. Genomewide analysis of TCP transcription factor gene family in Malus domestica. J. Genet. 93, 733-746.

Yao, X., Ma, H., Wang, J. \& Zhang, D.B., 2007. Genome-wide comparative analysis and expression pattern of TCP gene families in Arabidopsis thaliana and Oryza sativa. J. Integr. Plant Biol. 49, 885-897.

Zhou, M., Li, D., Li, Z., Hu, Q., Yang, C., Zhu, L. \& Luo, H., 2013. Constitutive expression of a miR319 gene alters plant development and enhances salt and drought tolerance in transgenic creeping bentgrass. Plant Physiol. 161, 1375-1391.

Zhou, Y., Xu, Z., Zhao, K., Yang, W., Cheng, T., Wang, J. \& Zhang, Q., 2016. Genome-wide identification, characterization and expression analysis of the TCP gene family in Prunus mume. Front. Plant Sci. 7, 1301. 